The Be Phenomenon in Early-Type Stars, IAU Colloquium 175

ASP Conference Series, Vol. 214, 2000

M. A. Smith, H. F. Henrichs, and J. Fabregat, eds.

\title{
A UV Spectrophotometric Technique for Measuring Temperatures of CS Structures over B Stars
}

\author{
Myron A. Smith \\ Catholic Univ. of America, Washington, DC
}

\section{Introduction: The Problem}

Do optical/UV variations of spectra of Be stars arise from pulsations or structures hanging over them? There is a need for a temperature-sensitive discriminator for circumstellar structures above Be stars to determine whether their UV flux variations are due to occulting structures or photospheric pulsations.

\section{Method of Attack}

To address this question, we acquired far-UV (SWP camera) echellograms covering $\lambda \lambda 1150-2100$ from the IUE final archive. For selected $B$ stars we have selected suitably exposed spectra observed within a short timespan, typically 13 days. We summed all the valid net fluxes within each of 60 echelle orders and placed them into an order vs. time array. We identify those times corresponding to high and low fluxes over several reference orders and form the quantity 1 $F_{\min }(\lambda) / F_{\max }(\lambda)$, for all echelle orders. The result is a low-resolution spectrum sensitive to absorptions at certain wavelengths, either strong lines such as Lyman $\alpha$ and several UV resonance lines or aggregates of metallic lines. All these are potentially sensitive to temperature variations.

We refer to the medium responsible for $F_{\text {min }}(\lambda)$ simply as a "reversing layer." We model this layer with two codes by I. Hubeny, SYNSPEC42, which computes the intensity spectra for a model atmosphere blanketed with $~ 100,000$ lines over the IUE/SWP wavelength range (Hubeny \& Heap 1996). A second code, CIRCUS, calculates the effects of a like number of lines (the "iron curtain") through a homogeneous cloud of specified parameters. The detailed cloud absorption spectrum is then binned to the wavelength grid of the IUE echelle orders for comparison with the observed absorption spectrum given above.

\section{Example of Computed Absorption Spectrum}

Grids of absorption spectra have been computed for various cloud temperatures, turbulences, and column densities. Figure 1 is an example, for which a density of $10^{23} \mathrm{~cm}^{-2}$ seems appropriate. The basic features of these spectra are that: (1) at shorter wavelengths the differences in absorption with $\mathrm{T}_{\text {cloud }}$ are greatest (mainly due to Ly $\alpha$ and Sill $\lambda 1206$ ) with the absorptions increasing most rapidly in the FUV for cool temperatures, and (2) the effects of the Si IV and CIV resonance doublets $(\lambda 1400, \lambda 1550)$ can be discerned in hotter cloud models. 


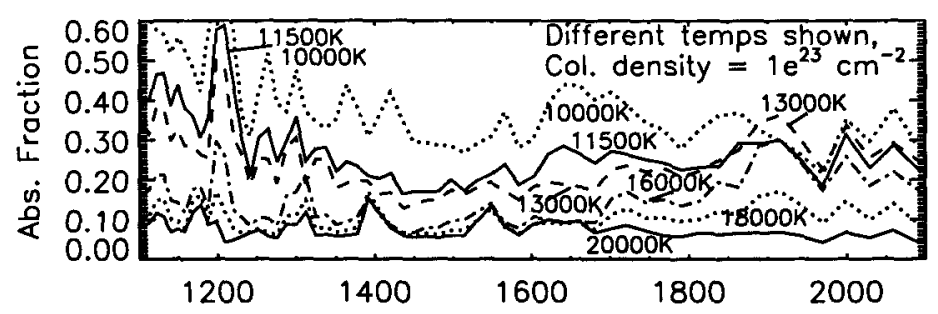

Figure 1. Cloud absorption spectra against $\mathrm{T}_{\mathrm{eff}}=22,000 \mathrm{~K}$ star

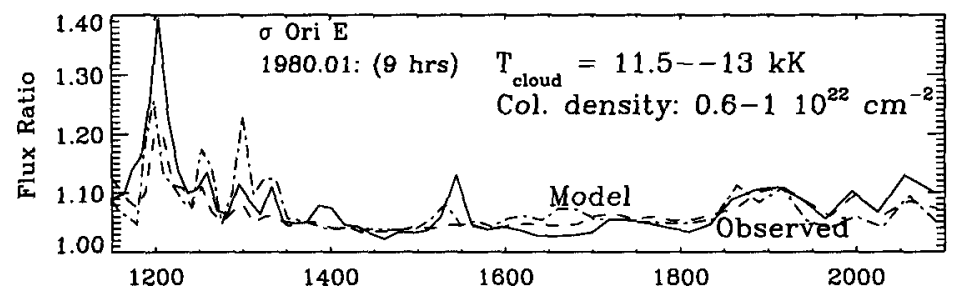

Figure 2. Fractional absorption spectrum for $\sigma$ Ori $\mathrm{E}$

\section{Proof of Concept: Examples of Magnetosphere and Pulsation}

As a test case, consider $\sigma$ Ori E, a magnetic-variable B2p star with a co-rotating magnetosphere (Short \& Bolton 1994). Figure 2 shows a comparison of a total of four high- and low-flux IUE spectra in 1980.01 with a model generated with $\mathrm{T}_{\mathrm{rev}}=13,000 \mathrm{~K}$ rmal metals, $1 / 2$ areal coverage $)$ and $11,500 \mathrm{~K}(1 / 10$ metals, full coverage, col. dens. $\left.=6 \times 10^{22} \mathrm{~cm}^{-2}\right)$. The absorptions of the model spectrum are shifted by +1 to compare like quantities. (There is a trade off between the areal coverage and column density; error in the coverage factor is set mainly by the uncertainty of the column density.) This example shows that this technique can work for the case of a homogeneous cloud.

As a second case, we examine in Figure 3a (top panel) flux ratios for four pulsating B stars, BW Vul, $\beta$ Cep, $\epsilon$ Per (NRP), and $\tau$ Her (NRPs). Their spectral ratios show common features: increasing absorption below $\lambda 1250$, a pair of resolved "windows" centered at $\lambda 1300$, a plateau in the range $\lambda \lambda 1350$ 1525 , and a gray to decreasing absorption at long wavelengths. We take these as empirical signatures of spectra as those produced in a single photosphere with a variable $T_{\text {eff }}$ during a pulsation cycle.

\section{The More Complicated Case of Pulsation}

Because stellar flux is formed over many photospheric layers, a ratio of hotphase/cool-phase spectra for a pulsator cannot be simply compared to the simple case of a homogenous reversing layer. Indeed, no single cloud absorption model we compute can simulate the pulsation signatures just noted. A better, but still imperfect, check for the pulsation case can be obtained by ratioing the 


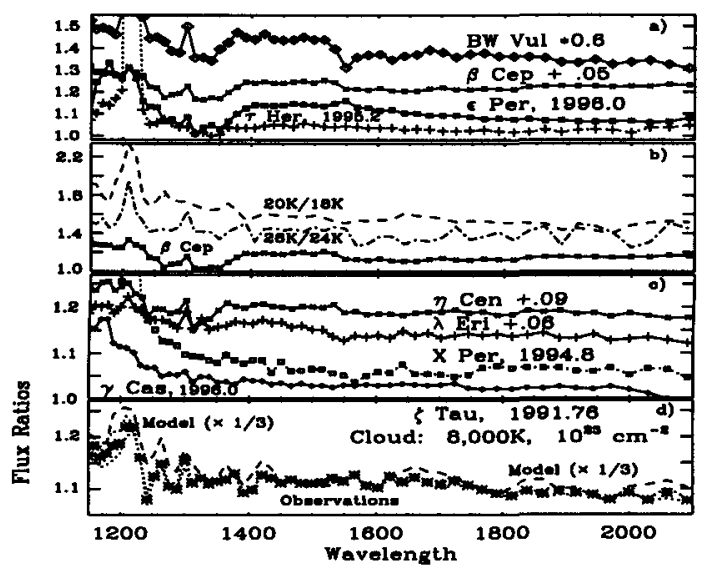

Figure 3. Fractional absorption spectra for top to bottom panels: a): four pulsators, $b$ : ratios of computed spectra for different $\mathrm{T}_{\text {eff }} \mathrm{S}$ and $\beta$ Cep, c): four Be stars of interest, $d$ ): zeta Tau (2 cycles)

fluxes of stellar atmospheres and binning them to the resolution of the IUE free spectral range. In Figure $3 \mathrm{~b}$ we show a spectrum ratio for $\beta$ Cep and the ratios of fluxes for $24,000 \mathrm{~K} / 22,000 \mathrm{~K}$ and $22,000 \mathrm{~K} / 20,000 \mathrm{~K}$ model atmospheres. The qualitative agreement is better than our homogeneous cloud-absorption results if one compares the observed absorptions with the flux ratios. Note the flat ratio at long wavelengths, the stronger absorption near $\lambda 1350$, and increasing absorption below $\lambda 1250$. Examination of opacity spectra computed for atmospheric densities and temperatures shows the reason for these signatures. There are no strong absorptions at long wavelengths. Absorptions from aggregates of excited lines and prominent or resonance lines of Ly $\alpha$, Si III, S III, and C III and their damping wings are responsible for the monotonic increase of absorption and hence decreased flux shortward of $\lambda 1250$ at lower values of $T_{\text {eff }}$. A multiple of Si III resonance lines combined with a near absence of lines at nearby wavelengths produces the double-window centered at $\lambda 1300$ in Fig. 3a.

\section{Results to Date}

Table 1 lists derived $T_{\text {rev }}$ values for spectral ratios examined for $23 \mathrm{~B}$ stars to date, based on comparison with computed absorptions of homogeneous clouds. Future work will modify and enlarge this table.

Because they are Be stars of particular interest, we consider results for $\lambda$ Eri, $\eta$ Cen, $\gamma$ Cas, and X Per. Note in Figure 3c that their absorptions increase monotonically with decreasing wavelength, a signature of absorbing layers with $T_{\text {rev }} \leq 10,000 \mathrm{~K}$. The first two stars show signatures consistent with 


\begin{tabular}{|c|c|c|c|c|}
\hline Star & $\mathrm{T}_{\text {eff }}$ & \# Ep./Sp. & $\mathrm{T}_{\text {rev }}$ Values & Comments \\
\hline X Per & 30 & $3 / 7$ & 10 & lg. col. dens. \\
\hline$\zeta \mathrm{Oph}$ & 30 & - & - & no vars. \\
\hline$\gamma$ Cas & 28 & $5 / 41$ & 15,10 & often 2 comps. \\
\hline $66 \mathrm{Oph}$ & 26 & $5 / 18$ & 10,18 & 2 temps. \\
\hline $2 \mathrm{Vul}$ & 26 & $3 / 13$ & 10,15 & 2 temps. \\
\hline$\epsilon$ Per & 24 & $2 / 91$ & 13,$18 ; 16$ & var. charact. \\
\hline$\alpha \mathrm{Vir}$ & 24 & $1 / 6$ & 15,18 & 2 comps. \\
\hline$\beta$ Cep & 24 & $9 / 43$ & $14.5-18$ & highly var., \\
\hline BW Vul & 24 & $7 / 37$ & $16-20$ & often 2 comps. \\
\hline$\lambda$ Eri & 24 & $7 / 46$ & $15-18$ & 1 or 2 comps., \\
\hline $28 \mathrm{CMa}$ & 22 & $1 / 2$ & 13 & \\
\hline$\omega$ Ori & 22 & $2 / 6$ & 12,16 & var. cool comp. \\
\hline$\eta$ Cen & 22 & $1 / 9$ & 14,17 & multi-comp? \\
\hline$\sigma$ Ori E & 22 & $3 / 11$ & $13-14.5$ & var. \\
\hline$\beta \mathrm{CMa}$ & 22 & $2 / 7$ & 16 & \\
\hline HD 37776 & 22 & $2 / 4$ & 13,16 & 2 comps. \\
\hline$\mu$ Cen & 22 & $2 / 9$ & 16 & gray \\
\hline$\alpha$ Eri & 20 & $2 / 10$ & 9 & \\
\hline$\zeta \mathrm{Tau}$ & 18 & $1 / 11$ & 8 & var. (cyc. to cyc.) \\
\hline EW Lac & 18 & $1 / 8$ & 10,14 & 2 comps. \\
\hline$o$ And & 16 & $1 / 4$ & 9 & \\
\hline$\theta \mathrm{CrB}$ & 16 & - & - & no vars \\
\hline$\tau \mathrm{Her}$ & 16 & $1 / 5$ & 9,13 & like EW Lac \\
\hline
\end{tabular}

$\mathrm{T}_{\text {rev }}=15,000-18,000 \mathrm{~K}$. This result is compatible with either pulsation or a cloud in radiative equilibrium with physical conditions near the star's surface. Layers with warm $\mathrm{T}_{\text {rev }}$ values may mean that the stars are pulsators or, like $\gamma$ Cas and HD 37776, have clouds whose temperatures are warm at the epoch of observation. This point needs to be kept in mind for objects observed only at one epoch (Table 1). In addition, $T_{\text {rev }}$ values appear to overlap for pulsators and magnetic stars at some times. In contrast, note in Figure 3d the unambiguously low value of $8000 \mathrm{~K}$ for $\zeta$ Tau. This figure suggests that this star has a cool corotating cloud (cf. Balona, these proceedings).

\section{Conclusions}

For stars with time-serial IUE data distributed over several epochs, spectrophotometric signatures provide temperature diagnostics that can be used to help decide whether an early-B star's variability is due to photospheric temperature modulation or an occulting CS structure. Such diagnostics are not sensitive to difficult-to-measure quantities such as stellar radius, which is otherwise a problem in clearly establishing the cause for line profile variability in Be stars.

\section{References}

Short, I. Bolton, C. 1994, IAU Symp. 162, eds. L. Balona, H.F. Henrichs, J.M. Le Contel, Eds., Kluwer, Dordrecht, p. 174

Hubeny, I., Heap, S. 1996, ApJ 470, 1144 